Information Engineering Express

International Institute of Applied Informatics

2019, Vol. 5, No. 2, $109-119$

\title{
An On-Demand Alternate Transportation Service System for Public Transportation using Real-Time Multi-agent Technology
}

\author{
Tomoichi Ebata *, Kojin Yano *, Yoshiyasu Takahashi *, \\ Tatsuhiro Sato ${ }^{\dagger}$
}

\begin{abstract}
For medium and large-scale urban rail services, any disruption of the operating schedule due to signal malfunction or accident involving injury or death can have wide ranging effects throughout the city. When such incidents occur, alternate means of transportation such as temporary bus services are arranged, but currently, operation of such buses is decided onsite. This research proposes a system that links agents in a multi-agent simulation to real-world transportation service companies and passengers and is able to make predictions within several minutes of an incident, of the operating state and transportation conditions 1 to 3 hours later, to decide a plan for operating alternate transportation based on these predictions, and to present this plan to bus companies and passengers in an on-line fashion. The results of simulations using a prototype of this system indicated that it will be able to facilitate bus companies to provide alternate transportation, and passengers to use such transportation, within five minutes of an incident occurring in a public transportation service on the scale of a city of one million people.
\end{abstract}

\section{Introduction}

Any disruption of the operating schedule of medium or large urban rail services due to signal malfunction or accident involving injury or death can have wide ranging effects throughout the city, and can take significant time to return to normal operation.

Current rail services have no way of knowing the locations or destinations of passengers when an accident occurs, and after the accident, they cannot predict what actions passengers will take, or the conditions resulting from their choices. For these reasons, it is difficult to predict passenger state in the future and create operating plans for temporary buses or other alternate transportation. It is also difficult to know ahead of time, whether provision and use of such alternate transportation will be beneficial to either the bus company or the passengers. Neither is it possible to initiate this sort of operating plan immediately after an accident occurs, to complete the plan before future effects of the incident occur, or to prepare such temporary buses before-hand.

In this research, we propose a system that is able to identify individual passengers, predict their

* Center for Technology Innovation - Systems Engineering, Hitachi, Ltd., Japan

$\dagger$ Service Platform Business Division, Hitachi, Ltd., Japan 
behavior and the state of the rail service after an incident, and to use the prediction to create an on-demand operating plan that will benefit both the bus companies and individual passengers. In particular, (1) agents in a multi-agent computer simulation (MAS) are generated and associated with real-world passengers and means of transportation (trains, buses), (2) immediately after a rail accident occurs, the computer simulation is used to predict the operating state of trains and traveling conditions for passengers one to three hours later, (3) independent operating rules are given to all bus and passenger agents in the simulation to predict their behaviors, and (4) these results are used to design an operating schedule for temporary buses in the real world.

Section 2 of this article describes objectives and issues regarding the on-demand alternate transportation scheduling system for urban public transportation discussed in this article. Section 3 describes related research. Section 4 describes the proposed system, and Section 5 reports on the results of simulations conducted using a prototype to evaluate the proposed system. Section 6 discusses conclusions and future prospects.

\section{Proposed System Objectives and Issues}

\subsection{Proposed System Objectives}

Objectives of the on-demand alternate transportation scheduling system are as follows.

(1) To plan temporary bus routes after rail accidents occur

When a railway signal malfunction or accident involving human injury or death occurs and particular sections of rail cannot continue operation, the state of damage after the incident must be estimated quickly, and temporary bus routes to minimize this damage must be drafted.

(2) To decide on temporary bus operation schedules that benefit both the bus companies and the passengers

Using the routes determined by the previous objective, an operating schedule that benefits the bus companies actually providing temporary bus services as well as the passengers must be created.

(3) To decide temporary bus operation schedules within five minutes of an accident

Considering that it takes an average of 70.2 minutes, with a standard deviation of 38.9 minutes, from a rail accident involving human injury or death until normal operation resumes, and considering the need for online inquiries to bus companies and passengers and time to prepare the temporary buses, the previous two operations must be completed within five minutes.

\subsection{Assumptions Regarding Applicable Public Transport Systems}

The following assumptions are made regarding public transport systems dealt-with in this research.

(1) Public transport systems for municipalities on a scale of one million people

This system deals with rail and bus systems in urban areas with population on a scale from several hundred thousand to one million. One hundred or more train stations, multiple rail companies with 100 or more trains operating, and up to 120,000 passengers using the rail system at a given time are assumed. Within this system, it is assumed temporary buses can be provided at roughly 30 train stations. 
(2) Knowing where passengers enter and exit the system

We assume that the times and locations where passengers enter and exit the public transport system can be known from IC card readers at automatic ticket gates. We also assume that the proposed system is able to use commuting data and past route data from passengers with commuter passes.

(3) Knowing accident conditions

We assume that the railway operations management systems are able to determine the time and scale of an accident on-line and can use information from past incidents to predict when operation will resume, and that this information can be used by the proposed system. Such assumptions are not necessary for the bus operations management systems.

\subsection{Tasks of the Proposed System}

(1) Obtain the current locations and destinations of rail system passengers

To provide temporary buses or other alternate means of transportation to train passengers, the current locations and final destinations of all current passengers must be obtained.

(2) Predict the operating state of the rail system after an accident

Operation of temporary buses must commence within a fixed period of time after an accident occurs, so the future operating state of the rail system, rather than the current state, must be predicted accurately, and operating schedules for multiple temporary buses must be planned.

(3) Suggest possible future passenger states after an accident and predict their behavior

Generally, after a railway accident, passengers must decide what they will do without having access to adequate information. It is difficult for an external system to predict these sorts of decisions. As such, our proposed system must provide quantitative predictions of whether passengers will use a temporary bus or not, and predict which choice each passenger will make.

(4) Complete the above tasks within a set period of time

The system must estimate the current and future states of several tens of thousands of passengers and create operating schedules for temporary buses between 100 or more stations within five minutes of an accident. The system must also decide on temporary bus routes and schedules that are beneficial to both the bus companies providing the temporary buses and all passengers, having inquired on-line whether such services can be provided by the companies and used by the passengers. All of this must be completed within ten minutes of the accident.

\section{Related Research}

There has been research mathematical models and algorithmic planning of train diagrams [1] [2] [3]. There has been research on multi-purpose optimization models for passenger train scheduling problems on railway networks, including multiple platforms [4] [5]. There has also been research implementing a high-speed simulation of tourism in a large urban area using parallel computation and an efficient algorithm, which achieved speeds 300-times real-time with over ten million agents representing tourists [6], and there has research focusing the behaviors of transportation management company and passengers [7] [8] [9]. There is also research operating the real-time display method [10]. In other research on traffic routing systems, driver agents were used to compute driver characteristics and preferences, driving routes, departure and arrival times and relations with information service providers and other drivers [11]. There is also research conducting 
online simulation of the flow of pedestrians in public buildings. This simulator was able to simulate different environments by just replacing the simulation environment [12] [13] [14]. Other research has simulated human behavioral psychology during emergencies such as fires and earthquakes to support design of emergency exits and escape routes [15] [16].

None of the above research performed simulations with agents associated with vehicles or users operating in the real world. Neither are they able to predict the future with associations for both vehicles and passengers. At this point in time, no system is able to present online solutions to accidents that have occurred. As such, this related research does not provide solutions to the issues presented by the proposed system.

\section{Proposed On-demand Alternate Transportation Planning System}

\subsection{Approaches for Resolving Issues}

\subsubsection{Dynamic creation and deletion of passenger agents}

When a real passenger is detected by an automatic ticket gate or IC card reader, a passenger agent is generated in the memory space of the computer and associated with this passenger. The passenger agent moves, linked to the motion of a train or bus agent, and is deleted when detected by the ticket gate or IC card reader at the destination location. In this way, the simulation is scalable to the numbers of passengers in a city of a million people.

\subsubsection{Releasing and reestablishing associations between reality and agents when an accident occurs}

Immediately after an accident, the association between agents for trains, buses and passengers and reality is severed, and they begin operating independently. Based on predictions of the incident, train agents continue to operate, passenger agents move based on the shortest route to their destination, and temporary bus agents operate based on operating time and numbers of passengers. Predictions of the future based on these agents are used to plan operation of temporary buses, and predictions of the future which include these temporary bus plans are also made. Thus, agents in the proposed system provide multiple predictions of future events. Finally, when the temporary bus schedule is finalized, the associations between train, bus, and passenger agents and their real counterparts are reestablished.

\subsubsection{Provision of information to agents and building rules into agents}

The predictions made in Section 4.1.2 above could result in operation and use of temporary buses that is not necessarily beneficial to the bus company or to passengers. For this reason, the predicted-future results obtained as in 4.1.2 are expressed using the passenger and temporary bus agents in the computer simulation. An algorithm that uses behavioral rules to decide operation of the temporary buses and whether passengers will use them is built into each of the agents. This is used to determine whether a proposal is beneficial and reject it if not.

\subsubsection{Accelerating route computation and reducing the number of messages sent}

In the simulation, when accidents and temporary bus routes are added, the routes for tens of thousands of people must all be recomputed at once, and when computing a temporary bus route, hundreds of 
thousands of route computations must be done. To complete this within five minutes, route computations must be done at high speed. Further, to finalize the temporary bus schedule within ten minutes, inquiries must be made to determine the actual number of passengers and whether temporary buses are available at the actual bus company, so the number of messages needed to do so must be minimized.

\subsection{On-demand Alternate Transportation Planning System Configuration}

The following figure gives an overview of the configuration of the proposed system.

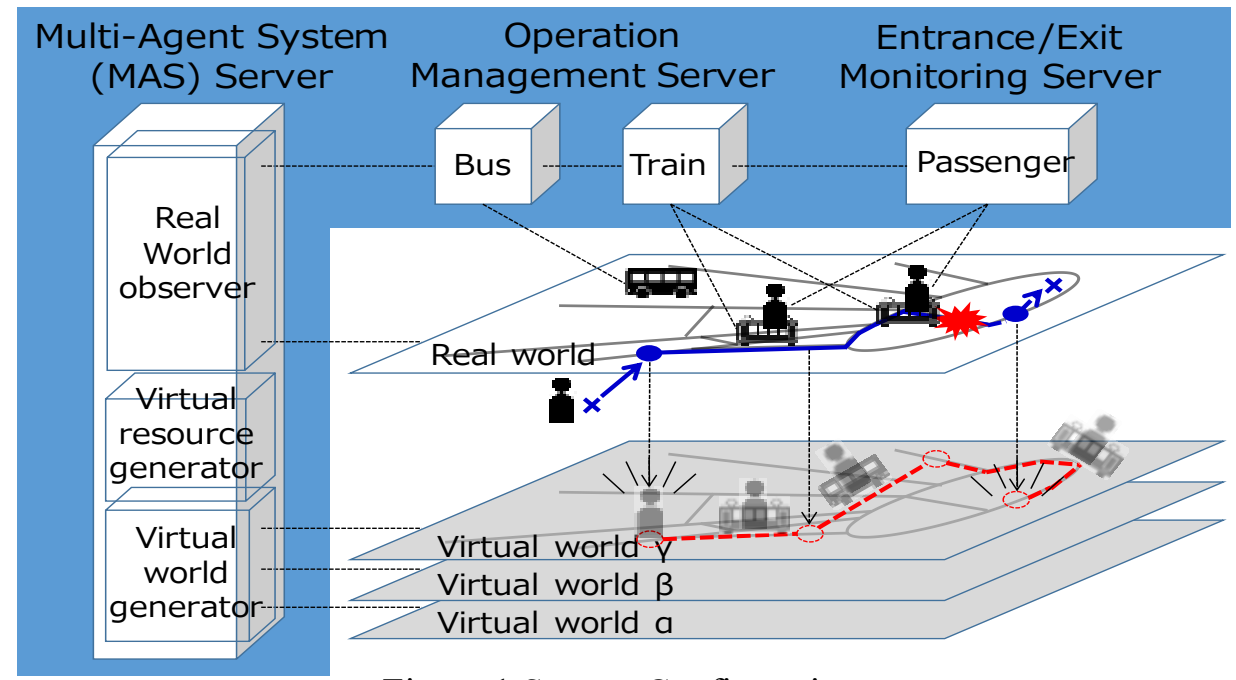

Figure 1:System Configuration

(1) Passenger entrance and exit management server

This server detects actual entrance and exit of passengers at stations and provides this information to the MAS server described below. It is also able to provide information regarding passenger commuter passes and past travel history.

(2) Railway operation management server

This server directs motion of trains and captures the numbers of passengers and real-time position of trains. When an accident is detected, it predicts when normal operation will be restored, and provides this information to the MAS server described below when necessary.

(3) Bus operations management server

This server directs operation of temporary buses and continuously captures the numbers of passengers and real-time positions of buses. It also maintains knowledge of the number of temporary buses available, their locations, and drivers, and provides information to the MAS server described below when necessary.

(4) Multi-agent simulation (MAS) server

The MAS server has three components. (a) The real-world observer generates and destroys MAS agents when real passengers enter or exit the system. It also applies the real-time position of real passengers and trains to these agents using information from the three servers described earlier. (b) The virtual world generator uses the MAS agents to predict conditions a set time in the future (1 to 3 hours) immediately after an accident occurs. Finally, (c) the virtual resource generator creates an optimal temporary bus operating plan based on predicted future states from (b). 


\subsection{On-demand Alternate Transportation Planning System Processing}

Processing performed by the proposed system after a rail accident is described below.

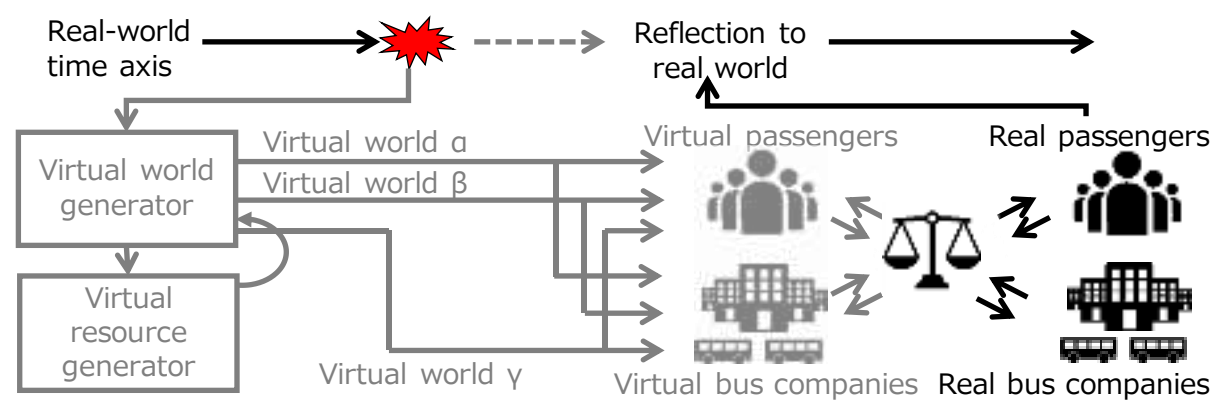

Figure 2: Outline of system processing

[Step 1] The railway operations management server detects the rail accident and estimates the time required before recovery.

[Step 2] The MAS server uses the passenger and train agents to predict the following in the near future (1 to 3 hours later). (a) The expected arrival time at the destination station for all passengers and (b) the number of passengers at every train station at a set time in the future (e.g.: $1 \mathrm{hr}$.) in (1) the world assuming the accident had not happened (Virtual world $\alpha$ ), and in the world assuming the incident did happen (Virtual world $\beta$ ). This step requires approximately two minutes.

[Step 3] The virtual resource generator in the MAS server uses the information from [Step 2] and a route search algorithm to compute a temporary bus operation plan (departure and arrival stations, number of buses, departure and arrival times). This step requires approximately one minute.

[Step 4] The MAS server uses the passenger and train agents to estimate (a) the expected arrival time at the destination station for all passengers using the temporary buses, and (b) the number of passengers at every train station at a set time in the future (e.g.: $1 \mathrm{hr}$.) in a virtual world assuming that temporary buses are used based on the operating plan from [Step 3] (Virtual world $\gamma$ ). This step requires approximately one minute.

[Step 5] The passenger and bus agents in the MAS system use the information in Virtual worlds $\alpha, \beta$, and $\gamma$ to decide the decision behavior of agents as follows. (a) Whether to board a temporary bus, and (b) what fare would be acceptable to pay when using the temporary bus. The figure below shows how these decisions are made (a fuzzy inference rule).

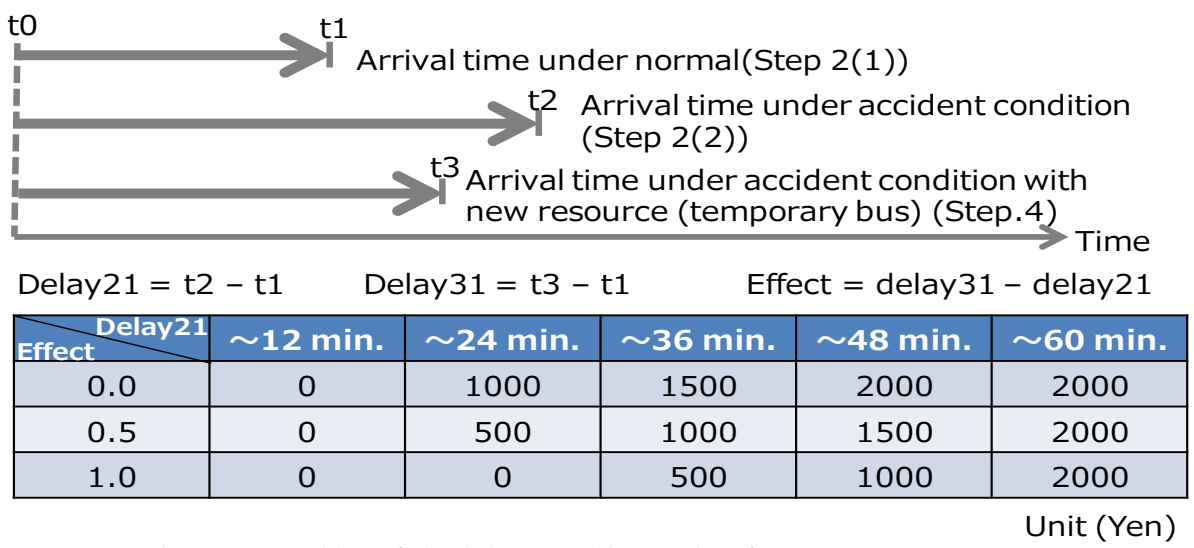

Figure 3: Table of decision making rules for passenger agents 
Bus agents in the MAS system use the number of passengers boarding and fare information computed above to decide whether they should operate or not. If the bus agent determines that operation would not be beneficial for the bus company, it decides not to operate that bus.

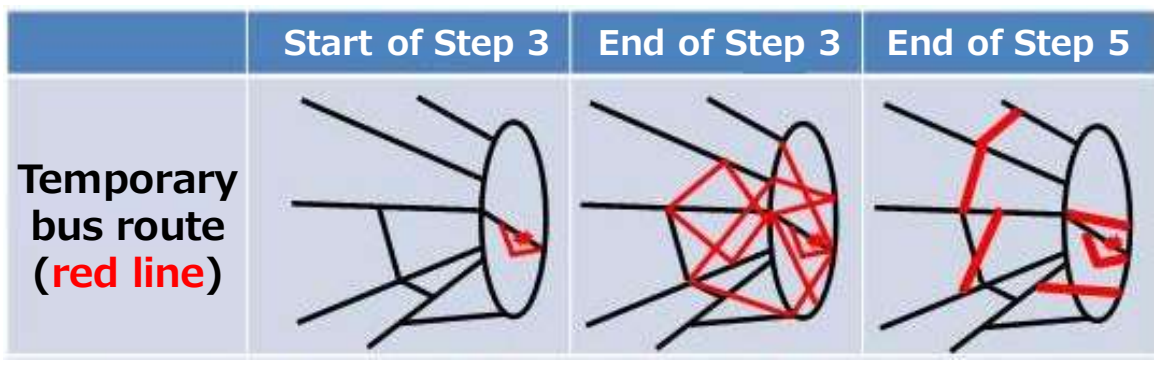

Figure 4: Temporary bus route creation process

With the above steps, a plan for providing and using temporary buses is completed between the bus agents and passenger agents in the MAS system. The time required for the steps to this point is a total of approximately five minutes.

[Step 6] The communications system of the passenger entry and exit management server is used to map the information from Virtual worlds $\alpha, \beta$, and $\gamma$ as described above, to the real-world buses and passengers linked to the agents, and to inquire whether the temporary buses will operate and whether they can be used. These inquiries must be done within five minutes.

[Step 7] Based on the results of these inquiries, the temporary bus schedule and passengers are decided, the bus companies are notified of the schedule, and passengers are notified of which bus they should take. In this way, operation of the temporary buses in the real world is decided and initiated within ten minutes of the accident.

\section{Prototype Verification}

\subsection{Prototype Overview}

\subsubsection{Computers used and server implementation}

All servers in Figure 1 were implemented on a single Windows 7 (64-bit) PC with an Intel Core ${ }^{\mathrm{TM}}$ CPU @ $3.4 \mathrm{GHz}$ and 16 GB RAM. The entry and exit events from the passenger entry and exit management server were simulated using population by time period from metropolitan Tokyo. For the rail operations management server, operating schedules from each of the rail companies as well as operation policies for when accidents occur were used. The messaging functions for sending inquiries to the actual bus companies and passengers were not implemented in the prototype.

\subsubsection{Acceleration of route search}

The proposed system must complete computation of temporary bus routes and passenger routes much more quickly than real time. As part of this research, we created algorithms using the wellknown Dijkstra and Warshall-Floyd algorithms, with constraints on the scope of route search, varied the numbers of passengers and stations in the models described above, and compared the computing times for the two algorithms.

The overview of the algorithm to constrain the scope of route search is given below. A provisional temporary route is first created by selecting the closest station to the incident (this is called a main 
station) and the nearest station on the same line or a different station that is within $4.5 \mathrm{~km}$ of the main station. This is given a score using a linear equation with variables for the number of passengers boarding, the average time saved per passenger, and the average rate at which passengers elect to take the bus. A hill-climbing method is then applied to search for the route with the highest score.
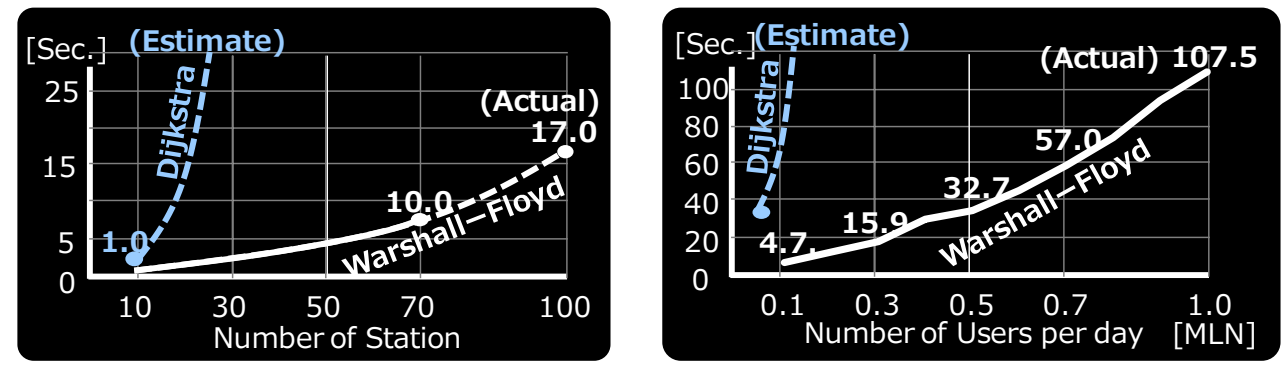

Figure 5 Calculation time results

Dijkstra's method is known as the fastest at computing the shortest route from a single starting node, but for the proposed system we used the Warshall-Floyd method, which finds the shortest routes between all pairs of nodes. The Warshall-Floyd method also has the feature that the results of all previous route searches can be used when searching for a new route.

\subsubsection{Agent behavior rules}

Weighted rule-based inference (fuzzy inference) was used as the algorithm for deciding the behavior of passenger agents (Step 5 above). Reasons for this choice include low computational load, the ability to describe rules in language, and the ability to tune it easily.

\subsection{Simulation Results}

The results of simulation using the prototype with a railway model are described below.

\subsubsection{Items to be verified}

Passenger positions and destinations when an accident occurs were implemented in the prototype as simulated events, and we used the MAS to get an idea of its capabilities for predicting future state of trains and passengers after an accident occurs. With this simulation we wanted to verify (1) whether temporary bus routes could be planned after a rail incident occurred, (2) whether bus schedules that are beneficial to both bus companies and passengers could be created, and (3) whether such temporary bus schedules could be created within five minutes of an accident.

\subsubsection{City model, railway system and MAS agents used}

The model of railway lines shown in the figure below was used in the prototype. This model was created by combining features from 67 municipal railway system maps in Japan.

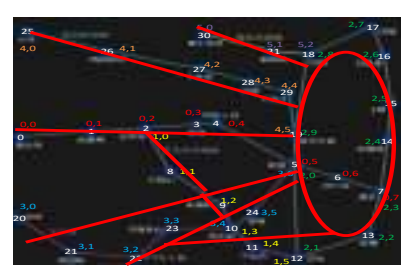

(a) Form of railway map

\begin{tabular}{|l|c|}
\hline (1) Passengers & $0.7-1.0$ million \\
\hline $\begin{array}{l}\text { (2) Bus companies } \\
\text { (Branch office) }\end{array}$ & $20-30$ \\
\hline (3) Stations & $70-100$ \\
\hline (4) Types of railway & $\begin{array}{l}\text { Ring 1, Branch 6, } \\
\text { Sub-branch 3 }\end{array}$ \\
\hline
\end{tabular}

(b) Numbers

Figure 6: Simulation railway system model 
We set the operating hours of service to from 06:00-26:00, and made from 1 to 5 temporary buses available at 30 specific train stations. The number of passengers varied according to population fluctuation data. For the accident, we made one line impassable at approximately $2 \mathrm{~km}$ from the station with the most passengers during the 90-minute period starting at $11 \mathrm{am}$.

The location data for MAS agents was updated 1,000 times an hour (every $3.6 \mathrm{~s}$ ). Note that the maximum number of passenger and train agents updated at the same time was approximately 120,000, which occurred in the morning between 7:30 and 8:00.

\subsubsection{Verifying temporary bus route planning after a rail accident}

The figure below shows the results of creating temporary bus routes in Step 3, as described in Section 4.3.
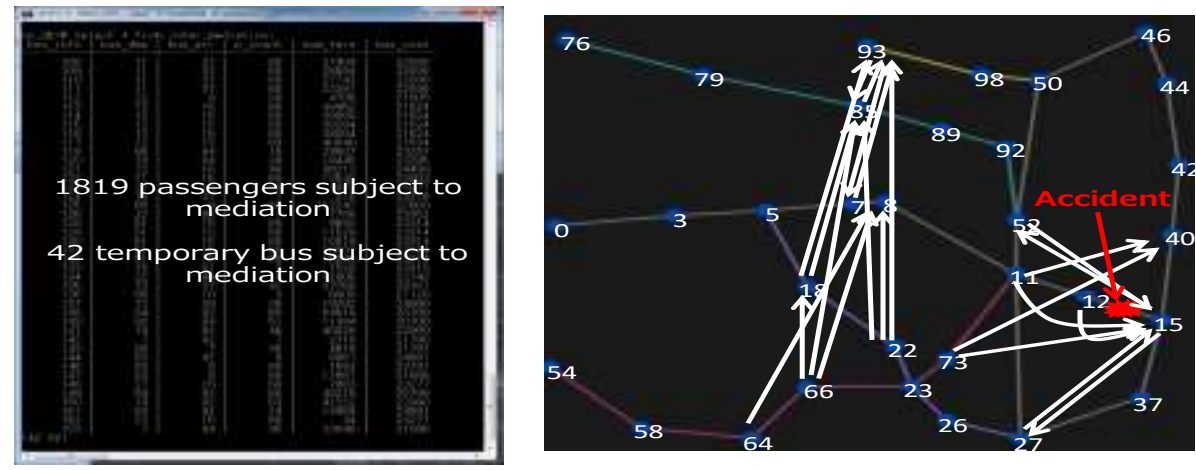

Figure 7: Proposed temporary bus route results

Due to the effects of the accident between stations 12 and 15, temporary buses were planned for most of the surrounding stations. Meanwhile, the effects of the accident spread to the end of a branch line (Station 11 in the figure), making this station a bottleneck, so temporary bus routes between stations straddling this branch line were also planned. This shows that in addition to locations near the accident, the temporary bus operating plan anticipated spread of the effects of the accident.

\subsubsection{Planning a temporary bus schedule that benefits both the bus company and the passengers}

The figure below shows the results of behavior of both passenger and bus agents, based on their own behavioral rules from Step 5, in the temporary bus operating plan described above.
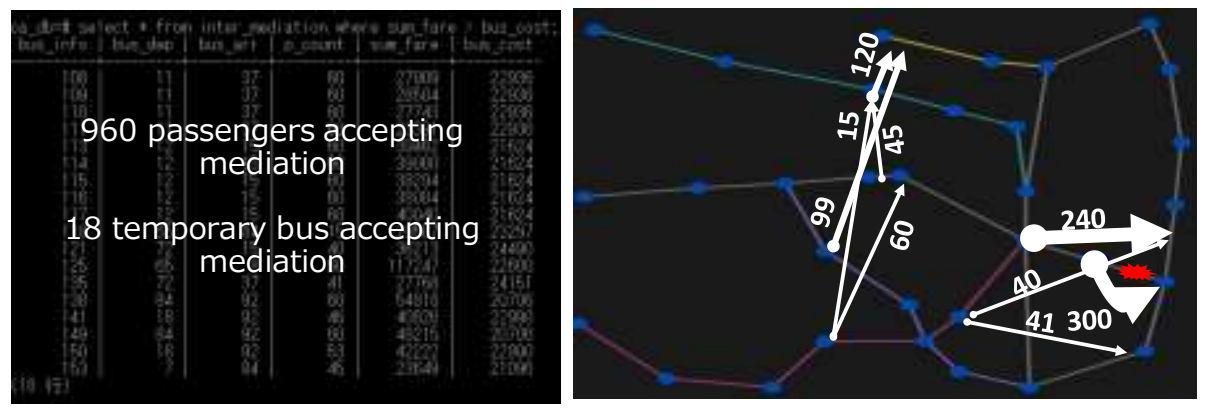

Figure 8: Numbers of passenger agents

This shows that before applying the agent behavior rules, 42 temporary buses were planned, and 1819 passengers used them, enabling them to arrive an average of 6.9 minutes earlier, but after 
applying the behavior rules, only 18 temporary buses were planned, 960 passengers used them, and they arrived an average of 21.3 minutes earlier. It also shows that revenue for the bus company changed from a loss of 18,337 yen/hour to income of 320,232 yen/hour. Further, although the transport capacity of the temporary buses was not more than $1.2 \%$ that of the railway at the time of the accident $(69,500$ passengers), congestion in train stations was reduced by $13 \%$.

\subsubsection{Verifying a temporary bus schedule was set within five minutes of the accident}

We were able to verify that a temporary bus schedule can be created in 2.9 minutes (174 s) assuming that approximately $70 \%$ of the population in a city of one million are commuters, and even if all one million residents commute, the schedule can be created in 5.3 minutes ( $315 \mathrm{~s}$ ).

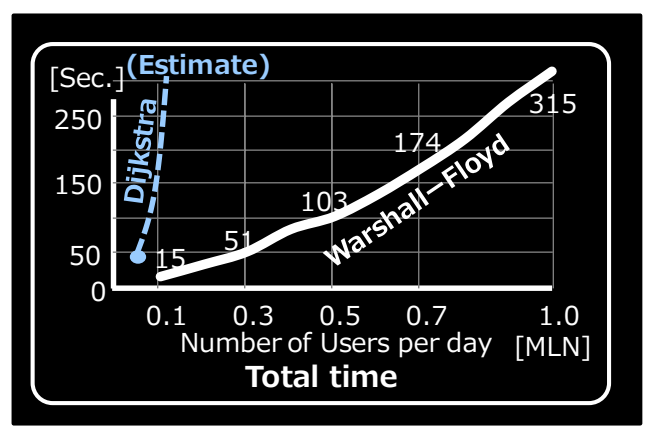

Figure 9: Total time

\section{Conclusion}

We have proposed a system that is able to make predictions within several minutes, of operating state and transport conditions one to three hours in the future, by associating agents in a multiagent simulation with transportation services and passengers in the real world. It can then use these predictions to create an on-demand temporary bus schedule that anticipates these predictions, and provides online suggestions to the bus company for operating the temporary buses, and to the passengers for using the temporary buses.

We verified the system using a prototype and confirmed that the system is able to create a plan for operation and use of alternate transportation, for the bus companies and passengers, within five minutes of an accident occurring in a public transport service in a city of one million people.

Note that we assumed a city of one million when studying this on-demand alternate transportation service, but it would be difficult to create such a plan for a city larger than this. To resolve this issue will require study of methods using super computers or parallel computation algorithms.

To verify that the agents incorporating behavioral rules in the MAS server actually behave similarly to buses and passengers in the real world will also require more practical testing. However for practical reasons, it would be difficult to conduct such tests in a city of one million, so it may be necessary to test in a smaller town of several thousand, or to add new functionality to revise behavioral rules automatically.

\section{References}

[1] Malachy Carey,David Lockwood, "A Model, Algorithms and Strategy for Train Pathing", 1995, Journal of the Operational Research Society, Issue 8 Volume 46. 
[2] JF Cordeau, P Toth, D Vigo, "A survey of optimization models for train routing and scheduling", 1998, Transportation Science, Volume 32, Issue 4 Pages 306-404.

[3] A Caprara, M Fischetti, P Toth, "Modeling and solving the train timetabling problem", 2002, Operations Research, Vol. 50, No. 5, Pages 851-861,

[4] Ghoseiri, K., Szidarovszky, F., Asgharpour, M.J., "A multi-objective train scheduling model and solution", 2004, Transportation Research Part B, 38, pp. 927-952.

[5]Yu-Hern Chang, Chung-Hsing Yeh, Ching Cheng Shen, "A multiobjective model for passenger train services planning: application to Taiwan's high-speed rail line", 2000, Transportation Research Part B: Methodological, Volume 34, Issue 2, Pages 91-106.

[6] Michael Balmer, Kai Nagel, Bryan Raney, " Large-Scale Multi-Agent Simulations for Transportation Applications", Intelligent Transportation Systems, 2004

[7] N Cetin, K Nagel, B Raney, A Voellmy, "Large-scale multi-agent transportation simulations", 2002, Computer Physics Communications, Volume 147, Issues 1-2, Pages 559-564.

[8] Raney, B.; Nagel, K., "An improved framework for large-scale multi-agent simulations of travel behaviour", 2006, Towards better Performing Transport Networks, Pages 305-347.

[9] Andreas Schadschneider, Hubert Klupfel, Tobias Kretz, Christian Rogsch, Armin Seyfried, "Fundamentals of Pedestrian and Evacuation Dynamics", 2009, "Multi-Agent Systems for Traffic and Transportation Engineering(IGI Global), Pages 124-154.

[10] AJ Heppenstall, AT Crooks, LM See, M Batty, "Methods, Techniques and Tools for the Design and Construction of Agent-Based Models", 2011, "Agent-based models of geographical systems(Springer), Pages 219-410.

[11] Jeffrey L Adler, Victor J Blue, "A cooperative multi-agent transportation management and route guidance system", Transportation Research Part C: Emerging Technologies. 10. pp. 433454.

[12] Andre Hanisch, Juri Tolujew, Klaus Richter, Thomas Schulze, "Modeling people flow: online simulation of pedestrian flow in public buildings", Proceedings of the 2003 Winter Simulation Conference, pp. 1635-1641.

[13] Nebi Sumer, "Personality and behavioral predictors of traffic accidents: testing a contextual mediated model", 2003, Accident Analysis \& Prevention, Volume 35, Issue 6, Pages 949-964.

[14] Emilio Moyano Diaz, "Theory of planned behavior and pedestrians' intentions to violate traffic regulations", 2002, Transportation Research Part F: Traffic Psychology and Behaviour, Volume 5, Issue 3, Pages 169-175.

[15] X. Pan, C. S. Han, K. Dauber, and K. H. Law, "A multi-agent based framework for the simulation of human and social behaviors during emergency evacuations," 2007, Ai \& Society, vol. 22, pp. 113-132.

[16] E.Ben-Elia, D Ettema, "Rewarding rush-hour avoidance: A study of commuters' travel behavior", 2011, Transportation Research Part A: Policy and Practice, Volume 45, Issue 7, Pages 567-582. 